\title{
A new approach on the stability analysis in ELKO cosmology
}

\author{
A. P. dos Santos Souza ${ }^{1, a}$, S. H. Pereira ${ }^{1, b}$, J. F. Jesus ${ }^{2, c}$ \\ ${ }^{1}$ Departamento de Física e Química, Faculdade de Engenharia de Guaratinguetá, UNESP-Univ. Estadual Paulista "Júlio de Mesquita Filho", \\ Av. Dr. Ariberto Pereira da Cunha 333-Pedregulho, Guaratinguetá, SP 12516-410, Brazil \\ ${ }^{2}$ Universidade Estadual Paulista “Júlio de Mesquita Filho", Campus Itapeva Rua Geraldo Alckmin 519, Vila N. Sra. de Fátima, Itapeva, \\ SP 18409-010, Brazil
}

Received: 29 August 2014 / Accepted: 5 January 2015 / Published online: 29 January 2015

(C) The Author(s) 2015. This article is published with open access at Springerlink.com

\begin{abstract}
In this work it has been developed a new approach to study the stability of a system composed by an ELKO field interacting with dark matter, which could give some contribution in order to alleviate the cosmic coincidence problem. It is assumed that the potential which characterizes the ELKO field is not specified, but it is related to a constant parameter $\delta$. The strength of the interaction between matter and ELKO field is characterized by a constant parameter $\beta$ and it is also assumed that both ELKO field and matter energy density are related to their pressures by equations of state parameters $\omega_{\phi}$ and $\omega_{m}$, respectively. The system of equations is analyzed by a dynamical system approach. It has been found the conditions of stability between the parameters $\delta$ and $\beta$ in order to have stable fixed points for the system for different values of the equation of state parameters $\omega_{\phi}$ and $\omega_{m}$, and the results are presented in form of tables. The possibility of decay of the ELKO field into dark matter or vice versa can be read directly from the tables, since the parameters $\delta$ and $\beta$ satisfy some inequalities. It allows us to constrain the potential assuming that we have a stable system for different interactions terms between the ELKO field and dark matter. The cosmic coincidence problem can be alleviated for some specific relations between the parameters of the model.
\end{abstract}

\section{Introduction}

Similarly to many segments in science, there are still open questions in modern cosmology to be answered. One of the greatest puzzles concerns the composition of the universe, which can be roughly divided into three components, namely the ordinary baryonic matter $(\approx 5 \%)$, the Dark Matter (DM) $(\approx 25 \%)$ and the Dark Energy $(\mathrm{DE})(\approx 70 \%)$, according to

\footnotetext{
a e-mail: alexandre.pinho510@gmail.com

b e-mail: shpereira@gmail.com

c e-mail: jfjesus@itapeva.unesp.br
}

the most accepted models [1-9]. Currently, we have direct access only to the former component, but there are many attempts to detect DM particles, since it behaves exactly like the usual baryonic matter, although not interacting electromagnetically [10-12]. DE is something even more mysterious, since their behavior is gravitationally repulsive [13-16]. From a theoretical point of view it is very common to interpret these entities as being some kind of field (scalar fields [17-27] or spinor fields [28-30], for example). Other models also consider the possibility of interaction between DM and DE [31-44], which could alleviate the coincidence problem for instance.

Besides the usual scalar fields, some recent works have shown that there are some classes of Non-Standard Spinors with interesting properties which could be useful in order to describe both DM and DE. One of such spinors is called ELKO, from German Eigenspinoren des Ladungsk onjugationsoperators, which has the property to be an eigenspinor of charge conjugation and parity, possessing nonlocality [45-62]. It satisfies (CPT) ${ }^{2}=-I$ and also has some other properties, like having spin $1 / 2$ and a mass dimension 1 , which makes it a good candidate to a particle with small probability of interacting with Standard Model particles, exactly as desired for the DM particles and maybe also to DE. The searching for ELKO dark matter at the CERN LHC has also been addressed recently $[63,64]$.

The ELKO spinor field, although being a fermionic field, can be factored out in a time dependent scalar field multiplied by a four components spinor field. Its scalar part drives the evolution of the pressure and energy density associated to the ELKO field, and it is through these physical quantities that ELKO contributes to cosmological equations. Here we will consider the ELKO field as a candidate to DE interacting with a DM field. The recent concern in this kind of exotic non-standard field as a good candidate to DE or DM is due to the fact that the scalar part of ELKO spinors has a much 
richer structure than the standard scalar field when applied to cosmology [17-27]. The coupled system of equations involving the interaction of DE and DM plus the Friedmann equations are much more involved, even for a standard scalar field $[26,27]$. Such a system is much more involved when dealing with ELKO fields. Due to its complexity, dynamical system analysis has been applied in order to deal with ELKO field as a possible candidate to DM or DE. Although exact solutions are not found even within this method for most cases, obtaining stability points that turn the system well behaved provides useful information as regards some undetermined parameters of the system. In our specific case we are interested in some conditions to be satisfied by the potential of the ELKO spinor field in order to keep the system stable. Maintaining system stability around some fixed points indicates that the involved system of equations has stable solutions for that specific region where the variables are evolving. In our case it can be interpreted as possible solutions where the ELKO field and DM can coexist, indicating a solution to the cosmic coincidence problem around these fixed points.

An interesting aspect concerning the ELKO field dynamics is that the choice of variables is an important question when one is looking for stable points of the dynamical system. Such a search for adequate variables depends in general on the structure of the physical quantities involved, like the energy density, pressure and Friedmann constraints. In some recent works [65-67], different choices of variables for interacting systems concerning the ELKO field have shown that there are no stable points in order to explain the cosmic coincidence problem. In [68] a new method of analysis was proposed, based on a constant parameter that leads to stable points under some conditions. In the present work the same method as [68] has been applied for a new set of dynamic variables and stable fixed points have been found for the system, including new interaction terms. This opens the possibility to alleviate the cosmological coincidence problem considering the ELKO field interacting with DM if the conditions found here are satisfied.

The new set of dynamical variables for the ELKO field used in this work is independent of the choice of potential. The potential dependence is set to a constant parameter $\delta$. Besides that it is assumed that the pressure and energy density of the ELKO field satisfy an equation of state of the form $p_{\phi}=\omega_{\phi} \rho_{\phi}$, where $p_{\phi}$ and $\rho_{\phi}$ are the ELKO field pressure and energy density, respectively. The dark matter content is described by an energy density $\rho_{m}$ that satisfies an equation of state of the type $p_{m}=\omega_{m} \rho_{m}$. There is also assumed an interaction between ELKO field and DM, characterized by a constant parameter $\beta$. The conditions for stability between $\delta$ and $\beta$ have been studied, depending on the type (radiation, dust, vacuum or ultrarelativistic matter) of the thermodynamic equation of state parameters $\omega_{m}$ and $\omega_{\phi}$.
Let us finish mentioning that the most general ELKO theory applied to cosmology should include torsion $[49,51,69$, 70]. This generalization is possible constructing an action where connections are no more symmetric at all. Such an antisymmetric part of the connection is defined as the torsion tensor, which is a rotation of a vector when it is parallel transported. Nevertheless problems with torsion have not been properly studied yet, although in some works we can see that torsion could play a cosmological constant role, accelerating the universe. It is also not well known if torsional effects appear to affect the dynamics only at the Planck scale.

The paper is organized as follows. In Sect. 2 we start presenting a general scenario including torsion and then we restrict ourselves to the torsionless case with the basic equations for a two fluid model, namely DM and ELKO field equations related to our cosmological applications in a spatially flat Friedmann-Lemaître-Robertson-Walker (FLRW) background. We present the pressure and energy density expressions, as well as the Friedmann equations and the conservation equations including the interaction between dark matter and ELKO field. We also define the variables concerning the dynamical system equations to be analyzed. Section 3 contains the main results. We study the stability of the dynamical system by imposing the restriction that the potential of the ELKO field is related to a constant parameter $\delta$. This analysis allows us to study the conditions to be satisfied by $\delta$ and $\beta$ for each equation of state parameters of DM and the ELKO field, in order to maintain stability. For each kind of interaction we present the results in the form of tables, specifying the equation of state parameter and conditions of stability to be satisfied. For DM and the ELKO field we restrict the equation of state parameters to vacuum, dust, radiation and ultrarelativistic matter. In Sect. 4 we conclude with some remarks.

\section{ELKO cosmology as a dynamical system}

We begin introducing the general Einstein-Cartan action:

$S=\int\left(\frac{1}{2 \kappa^{2}} \tilde{R}+\tilde{\mathcal{L}}_{\text {mat }}\right) \sqrt{-g} \mathrm{~d}^{4} x$

where the tilde denotes the presence of torsion terms in the covariant derivatives. We have also $\kappa^{2} \equiv 8 \pi G$ with the normalization $c=1$. The matter source (DM and ELKO) is present in the matter Lagrangian, $\tilde{\mathcal{L}}_{\text {mat }}$. In this work we have divided such a Lagrangian into two parts. One part concerns the DM (including baryonic matter), $\tilde{\mathcal{L}}_{\mathrm{DM}}$, with an attractive behavior. The other part is playing the role of a DE fluid, here represented by the ELKO spinor Lagrangian $\tilde{\mathcal{L}}_{\lambda}$ :

$\tilde{\mathcal{L}}_{\lambda}=\frac{1}{2} g^{\mu \nu} \tilde{\nabla}_{(\mu} \vec{\lambda} \tilde{\nabla}_{\nu)} \lambda-V(\vec{\lambda} \lambda)$. 
The covariant derivative acting on a spinor in the presence of torsion is

$\tilde{\nabla}_{a} \lambda=\partial_{a} \lambda-\frac{1}{4} \Gamma_{a} \lambda+\frac{1}{4} K_{a b c} \gamma^{b} \gamma^{c} \lambda$

where $\Gamma_{a}=\frac{1}{4} \Gamma_{a b c}\left[\gamma^{b} \gamma^{c}, \gamma^{b} \gamma^{c}\right]$, and $K_{a b c}$ is known as the contortion tensor, which represents the antisymmetric part of the Christoffel symbol. Then it is possible to find an analogous of the Einstein equation by variation of the action (1):

$\tilde{G}_{i j}=\tilde{R}_{i j}-\frac{1}{2} \tilde{R} g_{i j}=\kappa^{2} \Sigma_{i j}$,

and also an equation for the spin angular momentum tensor $\tau_{k}^{i j}$

$T_{k}^{i j}+\delta_{k}^{i} T_{l}^{j l}-\delta_{k}^{j} T_{l}^{i l}=\frac{\tau^{i j} k}{\kappa^{2}}$,

obtained by variation of the Einstein-Cartan action with respect to the contortion tensor $K^{i j}{ }_{k}$, which is related to the torsion by $T_{\mu \nu}^{\lambda}=\frac{1}{2}\left(K_{\nu \mu}^{\lambda}-K_{\mu \nu}^{\lambda}\right)$.

As well as in the ordinary Einstein equation, the right hand side of (4) represents the matter source, however, with a new form due to torsion. It is given by

$\Sigma_{i j}=\sigma_{i j}+\left(\tilde{\nabla}_{k}-K_{l k}^{l}\right)\left(\tau_{i j}{ }^{k}+\tau_{j}{ }_{i}{ }_{i}+\tau^{k}{ }_{i j}\right)$,

where $\sigma_{i j}$ corresponds to the energy momentum tensor related to $\tilde{\mathcal{L}}_{\text {mat }}$.

In a flat FLRW metric, $\mathrm{d} s^{2}=\mathrm{d} t^{2}-a(t)^{2}\left(\mathrm{~d} x^{2}+\mathrm{d} y^{2}+\mathrm{d} z^{2}\right)$, where $a(t)$ is the scale factor, the Christoffel symbols are $\Gamma_{t i}^{i}=\dot{a} / a$ and $\Gamma_{i i}^{t}=\dot{a} a$, where $i=x, y, z$, and

$\Sigma_{t t}=\frac{\tilde{\rho}}{2}, \quad \Sigma_{i i}=\frac{\tilde{p}}{2 a^{2}}$,

where $\tilde{\rho}$ and $\tilde{p}$ are the energy density and the pressure of the matter sources, in our case composed by DM and ELKO field, which can be approximated by a perfect fluid on large scales. If the ELKO field density depends only on cosmological time, as it must obey cosmological principle, the allowed components of the torsion tensor are reduced to

$T_{110}=T_{220}=T_{330}=h(t)$,

$T_{123}=T_{312}=T_{231}=f(t)$,

where $h$ and $f$ are functions carrying the torsion contributions. Finally, the Friedmann equations with torsion are given by

$H^{2}+2 h H+h^{2}-f^{2}=\frac{\kappa^{2}}{3} \tilde{\rho}$,

$\dot{H}-h H+\dot{h}-h^{2}+f^{2}=-\frac{\kappa^{2}}{2}(\tilde{\rho}+\tilde{p})$,

with $H=\dot{a} / a$.
From the above general treatment, we see that even assuming homogeneity and isotropy, the torsion functions $h(t)$ and $f(t)$ yet carry non trivial contributions to the time component of the evolution equations. Thus, even for an ELKO field that behaves dynamically as a scalar, its contributions should be taken into account. When it is used in cosmology, it is important to emphasize that they are spinors, nevertheless with important consequences: the most important of them is the fact that a totally coupled ELKO field must have torsion, and with torsion additional terms are present in the Friedmann equations, as seen above.

However, in order to study the possibility of ELKO field interaction with DM, intending to alleviate the cosmic coincidence problem, we will restrict our study to the torsionless case. It is evident that the presence of torsion should lead to more general results.

When the torsion terms are dropped out, it is easy to see that we obtain the ordinary Friedmann equation. Before doing this, let us just make a simplification on the ELKO field. As has been done in recent works [52-54,57,65], we will restrict the ELKO spinor field to the form $\lambda \equiv \phi(t) \xi$ and $\vec{\lambda} \equiv \phi(t) \vec{\xi}$, where $\xi$ and $\vec{\xi}$ are constant spinors. ${ }^{1}$ Then, in the torsion-free case, we have $h \rightarrow 0, f \rightarrow 0, \tilde{\rho} \rightarrow \rho_{m}+\rho_{\phi}$, and $\tilde{p} \rightarrow p_{m}+p_{\phi}$, where the subscripts $m$ and ${ }_{\phi}$ indicate, respectively, the DM and ELKO field contributions to the energy density and pressure.

Thus, the FLRW equations in a spatially flat background without torsion are given by

$$
\begin{aligned}
H^{2} & =\frac{\kappa^{2}}{3}\left(\rho_{m}+\rho_{\phi}\right), \\
\dot{H} & =-\frac{\kappa^{2}}{2}\left(\rho_{m}+p_{m}+\rho_{\phi}+p_{\phi}\right) .
\end{aligned}
$$

We will assume that the DM and ELKO field satisfy equations of state of the form $p_{m}=\omega_{m} \rho_{m}$ and $p_{\phi}=\omega_{\phi} \rho_{\phi}$, respectively. Due to the homogeneity of the field $\left(\partial_{i} \phi=0\right)$, the equation of motion that follows from (2) is substantially simplified to

$\ddot{\phi}+3 H \dot{\phi}-\frac{3}{4} H^{2} \phi+V_{, \phi}=0$,

where $V_{, \phi} \equiv \mathrm{d} V / \mathrm{d} \phi$. The pressure and energy density of the ELKO field are given by [54]:

$$
\begin{aligned}
p_{\phi} & =\frac{1}{2} \dot{\phi}^{2}-V(\phi)-\frac{3}{8} H^{2} \phi^{2}-\frac{1}{4} \dot{H} \phi^{2}-\frac{1}{2} H \phi \dot{\phi}, \\
\rho_{\phi} & =\frac{1}{2} \dot{\phi}^{2}+V(\phi)+\frac{3}{8} H^{2} \phi^{2} .
\end{aligned}
$$

\footnotetext{
${ }^{1}$ In [62] exact solutions have been presented to the ELKO spinor in spatially flat FLRW expanding spacetimes, and it has been shown that such factorization of the ELKO field time component is possible for some types of scale factors.
} 
The continuity equations for DM and ELKO field are:

$$
\begin{aligned}
\dot{\rho}_{m}+3 H\left(1+\omega_{m}\right) \rho_{m} & =Q, \\
\dot{\rho}_{\phi}+3 H\left(1+\omega_{\phi}\right) \rho_{\phi} & =-Q,
\end{aligned}
$$

where $Q$ stands for a possible interaction term between the $\mathrm{DM}$ and ELKO field. If $Q=0$, there is no interaction and both components evolve separately. If $Q>0$, the ELKO field decays into DM, an interesting scenario as regards inflation, and if $Q<0$, DM decays into the ELKO field (or DE).

Exact solutions to the system of coupled equations (14)(18) are not available. For this reason we make a stability analysis based on a dynamical system approach.

We define the new variables

$x=\frac{\kappa \dot{\phi}}{\sqrt{6} H}, \quad y=\frac{\kappa \sqrt{\tilde{V}}}{\sqrt{3} H}, \quad v=\frac{\kappa \sqrt{\rho_{m}}}{\sqrt{3} H}$,

where we have redefined the potential as $\tilde{V}=V+\frac{3}{8} H^{2} \phi^{2}$. The Friedmann equation (12) can be written as a constraint equation

$x^{2}+y^{2}+v^{2}=1$,

or in terms of the densities parameters, $\Omega_{\phi}+\Omega_{m}=1$, where

$\Omega_{\phi}=\frac{\kappa^{2} \rho_{\phi}}{3 H^{2}}=x^{2}+y^{2}, \quad \Omega_{m}=\frac{\kappa^{2} \rho_{m}}{3 H^{2}}=v^{2}$.

Equations (13), (17), and (18) can be written as a dynamical system of the form

$x^{\prime}=\frac{3}{2}\left(\omega_{m}-\omega_{\phi}\right) v^{2} x-\left[\frac{3}{2}\left(1+\omega_{\phi}\right)+\frac{\lambda}{2 H}\right] \frac{y^{2}}{x}-\frac{Q_{1}}{x}$,

$v^{\prime}=\frac{3}{2}\left(\omega_{\phi}-\omega_{m}\right)\left(1-v^{2}\right) v+\frac{Q_{1}}{v}$,

$y^{\prime}=\left(\frac{3}{2}\left(1+\omega_{\phi}\right)+\frac{3}{2}\left(\omega_{m}-\omega_{\phi}\right) v^{2}+\frac{\lambda}{2 H}\right) y$

where $\lambda=\frac{\dot{\tilde{V}}}{\tilde{V}}, Q_{1}=\frac{\kappa^{2} Q}{6 H^{3}}$, and' stands for the derivative with respect to $N \equiv \ln a$, such that $f^{\prime}=\dot{f} / H$ for any function $f$.

It is easy to see that the simple change of variables (19) should not be sufficient to rewrite the Friedmann equations (10) and (11) in a simple form with the torsion terms. At least two new variables would be necessary concerning $f$ and $h$, and also the system of Eqs. (22)-(24) would be much more complicated in the torsion case.

\section{Stability analysis}

The stability analysis of the above set of dynamical equations consists of finding fixed points $\bar{x}, \bar{v}$, and $\bar{y}$ that make $x^{\prime}, v^{\prime}$, and $y^{\prime}$ equal to zero. In the last section we have defined a three dimensional system according to our variable choice.
However, due to the Friedmann constraint (20) the system can be reduced to a two dimensional one.

Before we proceed, let us examine carefully the dynamical system (22)-(24). We see that, in addition to the dynamical variables $x, v$, and $y$ we also have the factor $\frac{\lambda}{2 H}=\frac{\tilde{V}^{\prime}}{2 \tilde{V}}$. The presence of the ' derivative shows that such term is also a dynamical variable, which should also be taken into account. However, $\tilde{V}$ depends on the potential, but the potential is not specified in our analysis, thus we cannot deal with this new variable. In order to avoid this problem, we set the additional assumption related to the potential, $-\frac{\lambda}{2 H} \equiv \delta$, where $\delta$ is a constant parameter. Such an imposition just reflects our ignorance on the potential $V(\phi)$.

Now it is easy to see that the resulting dynamical system, for a given interaction $Q_{1}(x, v, y)$, is written in terms of the dynamical variables $x, v, y$, and the constants $\omega_{m}, \omega_{\phi}$, and $\delta$. In order to analyze the stability of this system around the fixed points $\bar{x}, \bar{v}$, and $\bar{y}$ we must study the system satisfying $x^{\prime}=0$, $v^{\prime}=0$, and $y^{\prime}=0$. Notice that Eq. (24) is independent of the interaction $Q_{1}$, thus the condition $y^{\prime}=0$ can be achieved only if $\bar{y}=0$ or $-\frac{\lambda}{2 H}=\delta=\frac{3}{2}\left(1+\omega_{\phi}\right)+\frac{3}{2}\left(\omega_{m}-\omega_{\phi}\right) \bar{v}^{2}$. The first condition can be satisfied only if $\tilde{V}=0$ (see (19)), but from the definition of $\lambda$ this leads to a divergent $\lambda$. So, we restrict ourselves to the second condition, namely

$\delta=\frac{3}{2}\left(1+\omega_{\phi}\right)+\frac{3}{2}\left(\omega_{m}-\omega_{\phi}\right) \bar{v}^{2}$,

where $y^{\prime}=0$ even for $\bar{y} \neq 0$. By imposing the above condition on the dynamical system (22)-(24) we are left with a $2 \times 2$ system, since $y^{\prime}=0$ is always satisfied:

$\begin{aligned} x^{\prime}= & {\left[\frac{3}{2}\left(1+\omega_{\phi}\right)-\delta+\frac{3}{2}\left(\omega_{m}-\omega_{\phi}\right) v^{2}\right] x } \\ & +\left[\delta-\frac{3}{2}\left(1+\omega_{\phi}\right)\right] \frac{\left(1-v^{2}\right)}{x}-\frac{Q_{1}}{x},\end{aligned}$

$v^{\prime}=\frac{3}{2}\left(\omega_{\phi}-\omega_{m}\right)\left(1-v^{2}\right) v+\frac{Q_{1}}{v}$,

where we have also used the Friedmann constraint (20).

In order to study such a dynamical system it is worth to define its linearized matrix, with which one can determine the stability of a fixed point by just analyzing its determinant and trace. Such a mechanism is ensured by the HartmannGrobman theorem [71]. Thus, in the neighborhood of the fixed points we take infinitesimal displacements of the variables from the fixed points, $x \rightarrow \bar{x}+\delta x$ and $y \rightarrow \bar{v}+\delta v$, so that

$$
\left(\begin{array}{l}
\delta x^{\prime} \\
\delta v^{\prime}
\end{array}\right)=M\left(\begin{array}{l}
\delta x \\
\delta v
\end{array}\right)
$$

where $M$ is given by

$$
M=\left(\begin{array}{ll}
a & b \\
c & d
\end{array}\right)
$$


and

$a=-\left[\delta-\frac{3}{2}\left(1+\omega_{\phi}\right)\right] \frac{\left(1-\bar{v}^{2}\right)}{\bar{x}^{2}}+\frac{Q_{1}}{\bar{x}^{2}}-\frac{1}{\bar{x}} \frac{\partial Q_{1}}{\partial \bar{x}}$

$b=\frac{\partial x^{\prime}}{\partial v}=3\left(\omega_{m}-\omega_{\phi}\right) \bar{x} \bar{v}+\left[3\left(1+\omega_{\phi}\right)-2 \delta\right] \frac{\bar{v}}{\bar{x}}-\frac{1}{\bar{x}} \frac{\partial Q_{1}}{\partial \bar{v}}$,

$c=\frac{\partial v^{\prime}}{\partial x}=\frac{1}{\bar{v}} \frac{\partial Q_{1}}{\partial \bar{x}}$,

$d=\frac{\partial v^{\prime}}{\partial v}=\frac{3}{2}\left(\omega_{\phi}-\omega_{m}\right)\left(1-3 \bar{v}^{2}\right)-\frac{Q_{1}}{\bar{v}^{2}}+\frac{1}{\bar{v}} \frac{\partial Q_{1}}{\partial \bar{v}}$.

All variables carry a bar over them to show that the matrix $M$ is taken at the stable points which solve the system.

There is a simple way to know if the system described by the matrix $M$ is stable or not. It depends on the values of the determinant $(\Delta)$ and also on the trace $(\tau)$ of such matrix. When $\Delta>0$, both eigenvalues have the same sign and if they are positive, the solution increases with time, indicating that the solutions diverge from the fixed point and consequently this point is classified as unstable. On the other hand, when both eigenvalues are negative, the solution goes to zero and the fixed point is stable. In order to know what kind of fixed point we are dealing with, it is necessary to check the value of the trace of matrix $M$. When $\tau>0$, it means that both eigenvalues are greater than zero, describing unstable points. However, when $\tau<0$ we see that they are negative and the point is stable. For the case where $\Delta<0$ we see that both eigenvalues have opposite signs and then the fixed point is in fact a saddle point. Finally, when $\Delta=0$, at least one of the eigenvalues is zero and consequently nothing can be said about the stability of system.

Let us return to the dynamical system. Together with Eqs. (26) and (27), the assumption $-\frac{\lambda}{2 H}=\delta$ leads to the new constraint for the fixed point $\bar{v}$, according to (25):

$\bar{v}=\sqrt{\frac{2 \delta-3\left(1+\omega_{\phi}\right)}{3\left(\omega_{m}-\omega_{\phi}\right)}}$.

But it is easy to see that such a constraint already determines the value of the fixed point $\bar{v}$, since it depends only on the fixed parameters $\omega_{m}, \omega_{\phi}$ and $\delta$. Thus, in order to also satisfy the dynamical equation (27), we have verified that this restriction tells us that $Q_{1}$, which represents the interaction, could not assume an arbitrary value, since Eq. (27) would not be always solved for an arbitrary $Q_{1}$. In other words, we have found that when $Q_{1}$ does not depend on $x$, not all fixed $\bar{v}$ that make Eq. (27) vanish also satisfy (34), except for some very specific relations among the parameters $\omega_{m}, \omega_{\phi}$, and $\delta$. Such a restriction on the interaction term $Q_{1}$ is not so strong, since the variable $x$ is proportional to $\dot{\phi}$, which characterizes the time variation of the field $\phi$, which is reasonable for an interacting theory.
In the following, the stability conditions will be analyzed for different interaction terms between DM and ELKO field. The interaction terms are characterized by a dimensionless coupling constant $\beta$. We will search for the stability conditions between the parameters $\delta$ and $\beta$ for different equation of state parameters $\omega_{m}$ and $\omega_{\phi}$. Besides stability conditions characterized by negative eigenvalues of the matrix of perturbation $M$, we impose the additional reality condition on the parameters (19), namely we will impose $\bar{x}^{2}>0$, $\bar{v}^{2}>0$, and $\bar{y}^{2}>0$. As particular cases, we will discuss the physical content concerning the present time, characterized by $\bar{v}^{2}=\Omega_{m} \simeq 0.315, \bar{x}^{2}+\bar{y}^{2}=\Omega_{\phi} \simeq 0.685$, and $\omega_{m}=0$ according to recent observations based on the $\Lambda \mathrm{CDM}$ model [72]. We will also analyze the inflationary phase, where we believe there is no matter contribution, corresponding to $\bar{v}^{2} \rightarrow 0$.

\section{$3.1 Q_{1}=0$ and $Q_{1}=\beta$}

For the case $Q_{1}=0$ there is no interaction between the DM and the ELKO field, thus they evolve independently. The fixed points that follow from the analysis of the system (22) to (24) and satisfy $x^{\prime}=0, v^{\prime}=0$, and $y^{\prime}=0$ are given just by $\bar{x}=1, \bar{v}=0$, and $\bar{y}=0$, which does not represent a scaling solution, in the sense that does not admit a mixture of fluids. Besides that our model is valid just for $\bar{y} \neq 0$ and $\bar{v}$ given by (34).

For the case $Q_{1}=\beta$, a constant interaction term, we have scaling solutions of the form $\bar{x} \neq 0, \bar{v} \neq 0$, and $\bar{y}=0$, which could admit a mixture of the fluids, but the condition $\bar{y}=0$ shows that the potential part of the ELKO field is null according to (19), leading to $\tilde{V}=0$, and as discussed earlier, this leads to a divergence in the $\lambda$ term, but we have defined it as proportional to the constant $\delta$, so we will discard such a kind of fixed point in our analysis. We are interested only in fixed points that satisfy $\bar{x} \neq 0, \bar{v} \neq 0$, and $\bar{y} \neq 0$, which are scaling solutions and do not have null potential contributions.

$3.2 Q_{1}=\beta x^{2}$

Such an interaction between DM and ELKO field corresponds to $Q=\beta H \dot{\phi}^{2}$, where $\beta$ is a dimensionless parameter.

From the analysis of the system of Eqs. (26) and (27), the fixed points are given by $[\bar{x}, \bar{y}, \bar{v}]$, with

$$
\begin{aligned}
& \bar{x}=\sqrt{\frac{\left(3+3 \omega_{m}-2 \delta\right)\left(3+3 \omega_{\phi}-2 \delta\right)}{6 \beta\left(\omega_{\phi}-\omega_{m}\right)},} \\
& \bar{y}=\sqrt{\frac{\left(2 \delta-3 \omega_{m}-3\right)\left(2 \beta-2 \delta+3 \omega_{\phi}+3\right)}{6 \beta\left(\omega_{\phi}-\omega_{m}\right)}},
\end{aligned}
$$

and $\bar{v}$ is given by (34), which is valid for all interactions. 
Table 1 Stability conditions for some equation of state parameters of DM and ELKO field, corresponding to the interaction $Q_{1}=\beta x^{2}$

\begin{tabular}{|c|c|c|c|c|}
\hline & \multicolumn{4}{|l|}{ ELKO Spinor } \\
\hline & $\operatorname{Vacuum}\left(\omega_{\phi}=-1\right)$ & $\begin{array}{l}\text { Dust } \\
\left(\omega_{\phi}=0\right)\end{array}$ & $\begin{array}{l}\text { Radiation } \\
\left(\omega_{\phi}=1 / 3\right)\end{array}$ & $\begin{array}{l}\text { Ultrarelativistic } \\
\left(\omega_{\phi}=1\right)\end{array}$ \\
\hline \multicolumn{5}{|l|}{ Matter } \\
\hline Vacuum $\left(\omega_{m}=-1\right)$ & - & No stable point & No stable point & No stable point \\
\hline Dust $\left(\omega_{m}=0\right)$ & $\delta \leq 3 / 2$ if $\beta \geq 3 / 2$ or $\delta<\beta$ if $\beta<3 / 2$ & - & No stable point & No stable point \\
\hline Radiation $\left(\omega_{m}=1 / 3\right)$ & $\delta \leq \beta$ if $\beta \leq 2$ or $\delta<2$ if $\beta>2$ & $\begin{array}{l}\delta \leq 2 \text { if } \beta \geq 1 / 2 \\
\quad \text { or } \delta<3 / 2+\beta \\
\text { if } \beta<1 / 2\end{array}$ & - & No stable point \\
\hline Ultrarelativistic $\left(\omega_{m}=1\right)$ & $\delta \leq 3$ if $\beta \geq 3$ or $\delta<\beta$ if $\beta<3$ & $\begin{array}{l}\delta \leq 3 \text { if } \beta \geq 3 / 2 \\
\text { or } \delta<\beta+3 / 2 \\
\text { if } \beta<3 / 2\end{array}$ & $\begin{array}{c}\delta \leq 3 \text { if } \beta \geq 1 \text { or } \\
\delta<\beta+2 \text { if } \\
\beta<1\end{array}$ & - \\
\hline
\end{tabular}

The determinant $\Delta$ and the trace $\tau$ of the matrix of the linearized system (28) to (33) are given by

$$
\begin{aligned}
\Delta= & 4 \delta^{2}-2 \delta\left(2 \beta+6+3\left(\omega_{m}+\omega_{\phi}\right)\right)+6 \beta\left(1+\omega_{m}\right) \\
& +9\left(1+\omega_{m} \omega_{\phi}+\omega_{m}+\omega_{\phi}\right), \\
\tau= & 4 \delta-2 \beta-6-3\left(\omega_{m}+\omega_{\phi}\right) .
\end{aligned}
$$

The stability of the fixed points, namely $\Delta>0$ and $\tau<0$, is related with the values of $\delta, \beta, \omega_{m}$, and $\omega_{\phi}$. Table 1 presents the above conditions plus the reality conditions for the fixed points, namely $\bar{x}^{2}>0, \bar{v}^{2}>0$, and $\bar{y}^{2}>0$ for some specific values of the equation of state parameter for both DM $\left(\omega_{m}\right)$ and the ELKO field $\left(\omega_{\phi}\right)$.

Now let us analyze Table 1 . We are interested in two different epochs, namely the inflation and the late time acceleration epochs. The first one corresponds to an universe without DM and totally filled with the ELKO field. This means $\bar{v}^{2}=\Omega_{m}=0$ and $\omega_{m}=0$, which leads to $\delta=\frac{3}{2}\left(1+\omega_{\phi}\right)$. By replacing into $\bar{x}$ and $\bar{y}$ we obtain $\bar{x}^{2}=0$ and $\bar{y}^{2}=1$, which shows that all the contributions should come from the potential part $\tilde{V}$ and also we should have $\dot{\phi}=0$ from (19), but our interaction term $Q \sim \dot{\phi}$, thus we conclude that such a condition cannot be applied to inflation.

On the other hand, for the present time such variables are given by $\bar{v}^{2}=\Omega_{m}=0.315$ and $\bar{x}^{2}+\bar{y}^{2}=\Omega_{\phi}=$ 0.685 according to the $\Lambda \mathrm{CDM}$ model, and besides that we must have $\omega_{m}=0$. From (34) we conclude that: (i) $\delta \simeq$ 0.47 if $\omega_{\phi}=-1$-from the corresponding cell in Table 1 $\left(\omega_{\phi}=-1, \omega_{m}=0\right)$, we see that if $\beta \gtrsim 0.47$ the system is stable around the fixed points, and such a positive value of $\beta$ corresponds to decay of ELKO field into DM; (ii) $\delta \simeq 1.84$ if $\omega_{\phi}=1 / 3$, but there is no solution for $\beta$ from Table 1 in this case; and (iii) $\delta \simeq 2.53$ if $\omega_{\phi}=1$, which has no solution for $\beta$ too. We conclude that, for the present time, the only possibility in order to have stable fixed points is $\beta \gtrsim 0.47$, leading to decay of ELKO field into DM for an equation of state parameter $\omega_{\phi}=-1$, that is, the ELKO field behavior must be of vacuum type. Notice that other stability conditions are possible if the equation of state parameter of dark matter is of radiation or ultrarelativistic type.

The case $\omega_{m}=\omega_{\phi}$ has no physical meaning since both fluids have the same equation of state parameter; thus they are thermodynamically identical.

$3.3 Q_{1}=\beta v^{2} x^{2}$

For this interaction we have $Q=\frac{1}{3} \kappa^{2} H \beta \rho_{m} \dot{\phi}^{2}$. The fixed points are $[\bar{x}, \bar{y}, \bar{v}]$, with

$\bar{x}=\sqrt{\frac{3+3 \omega_{m}-2 \delta}{2 \beta}}$,

$\bar{y}=\sqrt{\frac{\left(2 \delta-3 \omega_{m}-3\right)\left(2 \beta-3 \omega_{m}+3 \omega_{\phi}\right)}{6 \beta\left(\omega_{\phi}-\omega_{m}\right)}}$,

and $\bar{v}$ is given by (34). From the linearized matrix one finds

$$
\begin{aligned}
\Delta= & \left(4-\frac{8 \beta}{3\left(\omega_{m}-\omega_{\phi}\right)}\right) \delta^{2}+\frac{1}{\omega_{m}-\omega_{\phi}} \\
& \times\left[\left(8+4 \omega_{m}+4 \omega_{\phi}\right) \beta-6\left(\omega_{m}^{2}-\omega_{\phi}^{2}+2 \omega_{m}-2 \omega_{\phi}\right)\right] \delta \\
& +\frac{1}{\omega_{m}-\omega_{\phi}}\left[-6 \beta\left(1+\omega_{\phi}+\omega_{m}+\omega_{\phi} \omega_{m}\right)\right. \\
& \left.-9\left(\omega_{\phi}-\omega_{m}+\omega_{\phi}^{2}-\omega_{m}^{2}+\omega_{m} \omega_{\phi}^{2}-\omega_{\phi} \omega_{m}^{2}\right)\right], \\
\tau= & \left(2-\frac{4 \beta}{3\left(\omega_{m}-\omega_{\phi}\right)}\right) \delta+\frac{1}{\omega_{m}-\omega_{\phi}}\left[2 \beta\left(1+\omega_{\phi}\right)\right. \\
& -3\left(\omega_{m}-\omega_{\phi}-\omega_{\phi}^{2}+\omega_{m} \omega_{\phi}\right) .
\end{aligned}
$$

Table 2 shows the stability conditions for some specific values of $\omega_{m}$ and $\omega_{\phi}$.

For the inflationary epoch $\left(\bar{v}^{2}=0\right.$ and $\left.\omega_{m}=0\right)$, we have the same condition for $\delta$, namely $\delta=\frac{3}{2}\left(1+\omega_{\phi}\right)$. From the corresponding cell in Table II it is easy to see that $\omega_{\phi}=-1$ is the only possible condition of stability, which leads to $\delta=0$, but such a value of $\delta$ is not possible from Table II if $\omega_{m}=0$.

For the present time we have $\bar{v}^{2}=\Omega_{m}=0.315$. As the previous case, from (34) we have: (i) $\delta \simeq 0.47$ if $\omega_{\phi}=-1$, 
Table 2 Stability conditions for some equation of state parameters of DM and ELKO field, corresponding to the interaction $Q_{1}=\beta v^{2} x^{2}$

\begin{tabular}{|c|c|c|c|c|}
\hline & \multicolumn{4}{|l|}{ ELKO Spinor } \\
\hline & Vacuum $\left(\omega_{\phi}=-1\right)$ & Dust $\left(\omega_{\phi}=0\right)$ & $\begin{array}{l}\text { Radiation } \\
\left(\omega_{\phi}=1 / 3\right)\end{array}$ & $\begin{array}{l}\text { Ultrarelativistic } \\
\left(\omega_{\phi}=1\right)\end{array}$ \\
\hline \multicolumn{5}{|l|}{ Matter } \\
\hline $\operatorname{Vacuum}\left(\omega_{m}=-1\right)$ & - & No stable point & No stable point & No stable point \\
\hline Dust $\left(\omega_{m}=0\right)$ & $\frac{18}{2 \beta+9}<\delta<\frac{3}{2}$ if $\beta>\frac{3}{2}$ & - & No stable point & No stable point \\
\hline Radiation $\left(\omega_{m}=1 / 3\right)$ & $\frac{16}{\beta+6}<\delta<2$ if $\beta>2$ & $\begin{array}{l}\frac{(6 \beta+13)}{4 \beta+6}<\delta<2 \\
\text { if } \beta>\frac{1}{2}\end{array}$ & - & No stable point \\
\hline Ultrarelativistic $\left(\omega_{m}=1\right)$ & $\frac{36}{\beta+9}<\delta<3$ if $\beta>3$ & $\begin{array}{c}\frac{3}{2} \frac{2 \beta+21}{2 \beta+9}<\delta<3 \\
\text { if } \beta>\frac{3}{2}\end{array}$ & $\begin{array}{l}\frac{2(\beta+5)}{\beta+3}<\delta<3 \\
\text { if } \beta>1\end{array}$ & - \\
\hline
\end{tabular}

which is possible if $\beta \gtrsim 14.6$ and implies a positive value of $\beta$, consequently the decay of ELKO field into DM. The other cases: (ii) $\delta \simeq 1.84$ if $\omega_{\phi}=1 / 3$, and (iii) $\delta \simeq 2.53$ if $\omega_{\phi}=1$ do not present stable solutions if $\omega_{m}=0$.

\section{$3.4 Q_{1}=\beta\left(x^{2}+y^{2}\right) x^{2}$}

For this interaction we have $Q=\frac{1}{3} \frac{\kappa^{2}}{H} \beta \rho_{\phi} \dot{\phi}^{2}$.

The fixed points are $[\bar{x}, \bar{y}, \bar{v}]$, with

$\bar{x}=\sqrt{\frac{2 \delta-3-3 \omega_{\phi}}{2 \beta}}$,

$$
\begin{aligned}
\tau= & \left(2+\frac{4 \beta}{3\left(\omega_{m}-\omega_{\phi}\right)}\right) \delta+\frac{1}{\omega_{m}-\omega_{\phi}}\left[-2 \beta\left(1+\omega_{m}\right)\right. \\
& \left.-3\left(\omega_{m}-\omega_{\phi}+\omega_{m}^{2}-\omega_{m} \omega_{\phi}\right)\right] .
\end{aligned}
$$

Table 3 shows the stability conditions for some types of $\omega_{m}$ and $\omega_{\phi}$.

For the inflationary epoch $\left(\bar{v}^{2}=0\right.$ and $\left.\omega_{m}=0\right)$ we have $\bar{x}^{2}=0$ and $\bar{y}^{2}=1$, which shows that the contribution comes only from the potential part. The kinetic part is null, but the interaction is proportional to $\dot{\phi}^{2}$; thus this interaction does not apply to inflation.

$\bar{y}=\sqrt{\frac{2 \delta\left(2 \beta+3 \omega_{m}-3 \omega_{m}\right)-6 \beta\left(1+\omega_{m}\right)+9\left(\omega_{\phi}^{2}-\omega_{m} \omega_{\phi}-\omega_{m}+\omega_{\phi}\right)}{6 \beta\left(\omega_{\phi}-\omega_{m}\right)}}$,

and $\bar{v}$ given by (34).

From the linearized matrix one finds

$$
\begin{aligned}
\Delta= & \left(4+\frac{8 \beta}{3\left(\omega_{m}-\omega_{\phi}\right)}\right) \delta^{2}-\frac{1}{\omega_{m}-\omega_{\phi}}\left[8 \beta\left(1+\omega_{m}\right)\right. \\
& \left.+6\left(\omega_{m}^{2}-\omega_{\phi}^{2}+2 \omega_{m}-2 \omega_{\phi}\right)\right] \delta \\
& +\frac{1}{\omega_{m}-\omega_{\phi}}\left[6 \beta\left(1+\omega_{m}^{2}+2 \omega_{m}\right)\right. \\
& \left.+27\left(\omega_{\phi} \omega_{m}^{2}+\omega_{m}^{2}-\omega_{\phi}^{2} \omega_{m}+\omega_{m}-\omega_{\phi}^{2}-\omega_{\phi}^{2}-\omega_{\phi}\right)\right],
\end{aligned}
$$

Considering the present time $\left(\bar{v}^{2}=0.315\right)$ we have: (i) $\delta \simeq 0.47$ if $\omega_{\phi}=-1-$ we have verified that for $\beta \gtrsim 0.69$ we have $\bar{x}^{2}>0$ and $\bar{y}^{2}>0$, which represents stable fixed points. The cases (ii) $\delta \simeq 1.84$ if $\omega_{\phi}=1 / 3$, and (iii) $\delta \simeq 2.53$ if $\omega_{\phi}=1$ do not present stable fixed points for $\omega_{m}=0$.

$$
3.5 Q_{1}=\beta\left(v^{2}-y^{2}\right)
$$

The corresponding interaction is $Q=2 \beta H\left(\rho_{m}-\rho_{\phi}+\frac{1}{2} \dot{\phi}^{2}\right)$. The fixed points are $[\bar{x}, \bar{y}, \bar{v}]$, with

$\bar{x}=\sqrt{\frac{4 \delta^{2}+2 \delta\left(4 \beta-6-3\left(\omega_{m}+\omega_{\phi}\right)\right)-6 \beta\left(2+\omega_{m}+\omega_{\phi}\right)+9\left(1+\omega_{m}+\omega_{\phi}+\omega_{m} \omega_{\phi}\right)}{6 \beta\left(\omega_{\phi}-\omega_{m}\right)}}$, 
Table 3 Stability conditions for some equation of state parameters of DM and ELKO field, corresponding to the interaction $Q_{1}=\beta\left(x^{2}+y^{2}\right) x^{2}$

\section{ELKO Spinor}

$\operatorname{Vacuum}\left(\omega_{\phi}=-1\right)$

Dust $\left(\omega_{\phi}=0\right)$

Radiation

$\left(\omega_{\phi}=1 / 3\right)$

Ultrarelativistic

$\left(\omega_{\phi}=1\right)$

Matter

Vacuum $\left(\omega_{m}=-1\right)$

Dust $\left(\omega_{m}=0\right)$

Radiation $\left(\omega_{m}=1 / 3\right)$

Ultrarelativistic $\left(\omega_{m}=1\right)$

$$
\begin{aligned}
& 0<\delta<\frac{3 \beta}{3+2 \beta} \text { if } \beta>0 \\
& 0<\delta<\frac{2 \beta}{2+\beta} \text { if } \beta>0 \\
& 0<\delta<\frac{3 \beta}{3+\beta} \text { if } \beta>0
\end{aligned}
$$

$$
\begin{aligned}
& \text { No stable point } \\
& - \\
& \frac{3}{2}<\delta<\frac{3+8 \beta}{2+4 \beta} \text { if } \\
& \beta>0 \\
& \frac{3}{2}<\delta<\frac{9+12 \beta}{6+4 \beta} \\
& \text { if } \beta>0
\end{aligned}
$$

No stable point
No stable point
-
$2<\delta<\frac{2+3 \beta}{1+\beta}$ if
$\beta>0$

No stable point

No stable point

No stable point

No stable point

$\beta>0$

$$
\bar{y}=\sqrt{\frac{\left(2 \delta-3-3 \omega_{\phi}\right)\left(2 \delta+2 \beta-3-3 \omega_{m}\right)}{6 \beta\left(\omega_{m}-\omega_{\phi}\right)}},
$$

and $\bar{v}$ given by (34).

The determinant and trace are given by

$$
\begin{aligned}
\Delta= & 4 \delta^{2}+2 \delta\left(2 \beta-6-3\left(\omega_{\phi}+\omega_{m}\right)\right)-6 \beta\left(1+\omega_{\phi}\right) \\
& +9\left(1+\omega_{m}+\omega_{\phi}+\omega_{m} \omega_{\phi}\right), \\
\tau= & 4 \delta-6+2 \beta-3\left(\omega_{m}+\omega_{\phi}\right) .
\end{aligned}
$$

Table 4 shows the stability conditions for some specific values of $\omega_{m}$ and $\omega_{\phi}$.

For the inflationary epoch $\left(\bar{v}^{2}=0\right.$ and $\left.\omega_{m}=0\right)$, we have the same condition for $\delta$, namely $\delta=\frac{3}{2}\left(1+\omega_{\phi}\right)$. Contrary to the previous cases, we see that $\omega_{\phi}=-1$ is not a stable solution. For $\omega_{\phi}=1 / 3$ we have $\delta=2$, which is a stable solution corresponding to $\beta=-1 / 2$, representing the decay of DM into the ELKO field. But we have inferred $\bar{v}^{2}=0$, thus there is no matter to decay at the inflation epoch. For $\omega_{\phi}=1$ we have $\delta=3$, but the stability condition requires $\delta<3$ from the corresponding cell in Table IV. We conclude that such an interaction does not present stable points for the inflation.

For the present time we have $\bar{v}^{2}=0.315$ and $\omega_{m}=0$. As in the previous case, we have: (i) $\delta \simeq 0.47$ if $\omega_{\phi}=-1$, which has no stable solution; (ii) $\delta \simeq 1.84$ if $\omega_{\phi}=1 / 3$, and it is easy to see from Table IV that such a value of $\delta$ is possible for a negative value of $\beta$ - for instance, if $\beta=-1 / 2$ the condition for $\delta$ is $1.69 \lesssim \delta \lesssim 2$; and (iii) $\delta \simeq 2.53$ if $\omega_{\phi}=1$, and for this condition we also have stable solution for a negative $\beta$, as can be seen from Table IV. If $\beta=-3 / 2$ for instance, the condition on $\delta$ is $2.07 \lesssim \delta \lesssim 3.0$, which includes $\delta \simeq 2.53$. Thus, contrary to the previous cases, if the ELKO equation of state parameter is of radiation or ultrarelativistic type, the system presents stable solutions if $\beta$ is negative, which corresponds to the decay of DM into ELKO field. The present acceleration of the universe can be understood in this model as the decay of dark matter into ELKO particles. This is a very interest- ing scenario which also alleviates the cosmic coincidence problem.

\section{Concluding remarks}

In this work we have developed a new approach to study the stability of a system composed by an ELKO field interacting with DM, which could give some contribution in order to alleviate the cosmic coincidence problem. Since recent works [65-68] have not found stable points for such system for different dynamic variables and interactions terms, we are led to believe (without demonstration) that the system ELKO-DM does not allow stable points. Based on these results, we have supposed there to be an additional constraint to the dynamical system, namely that the potential of the ELKO field is related to a constant parameter $\delta$; then we have analyzed the stability conditions for such a new system. We have also assumed that both the ELKO field and the dark matter energy density are related to the pressure by equations of state parameters $\omega_{\phi}$ and $\omega_{m}$, respectively. We have found different stability conditions relating the parameter $\delta$ and the interaction parameter $\beta$, which states if the decay is from DM to $\operatorname{ELKO}(\beta<0)$ or from ELKO to DM $(\beta>0)$. Different values of $\omega_{\phi}$ and $\omega_{m}$ corresponding to vacuum, dust, radiation, and ultrarelativistic equation of state parameter are presented in Tables 1, 2, 3, 4 for different interaction terms, showing the conditions for the existence of stable points.

For the first three tables, corresponding to the interactions $Q_{1}=\beta x^{2}, Q_{1}=\beta v^{2} x^{2}$, and $Q_{1}=\beta\left(x^{2}+y^{2}\right) x^{2}$, the conditions for stable fixed points in order to satisfy the present stage of acceleration (with $\omega_{m}=0$ ) are given by positive $\beta$ and $\delta$ parameters and also require an equation of state parameter for the ELKO field of vacuum type $\left(\omega_{\phi}=-1\right)$. Positive values of $\beta$ means the decay of ELKO field into DM particles. Such conditions could alleviate the cosmological coincidence problem. The inflationary phase cannot be driven for these interactions if we set $\omega_{m}=0$ and $\bar{v}^{2}=0$. Other pos- 
Table 4 Stability conditions for some equation of state parameters of $\mathrm{DM}$ and ELKO field, corresponding to the interaction $Q_{1}=\beta\left(v^{2}-y^{2}\right)$. We have defined the following parameters: $\delta_{1}=\frac{3}{4}-\beta-\frac{1}{4} \sqrt{9+16 \beta^{2}}$; $\delta_{2}=1-\beta-\sqrt{1+\beta^{2}} ; \delta_{3}=\frac{3}{2}-\beta-\frac{1}{2} \sqrt{9+4 \beta^{2}} ; \delta_{4}=\frac{7}{4}-\beta-$ $\frac{1}{4} \sqrt{1+16 \beta^{2}} ; \delta_{5}=\frac{9}{4}-\beta-\frac{1}{4} \sqrt{9+16 \beta^{2}} ; \delta_{6}=\frac{5}{2}-\beta-\frac{1}{2} \sqrt{1+4 \beta^{2}}$

\section{ELKO Spinor}

\begin{tabular}{|c|c|c|c|}
\hline $\begin{array}{l}\text { Vacuum } \\
\left(\omega_{\phi}=-1\right)\end{array}$ & Dust $\left(\omega_{\phi}=0\right)$ & $\begin{array}{l}\text { Radiation } \\
\left(\omega_{\phi}=1 / 3\right)\end{array}$ & $\begin{array}{l}\text { Ultrarelativistic } \\
\left(\omega_{\phi}=1\right)\end{array}$ \\
\hline
\end{tabular}

\section{Matter}

Vacuum
$\left(\omega_{m}=-1\right)$

Dust $\left(\omega_{m}=0\right)$

Radiation

$\left(\omega_{m}=1 / 3\right)$

Ultrarelativistic

$\left(\omega_{m}=1 / 3\right)$
No stable point

No stable point

No stable point

$$
\begin{aligned}
& \delta_{1}<\delta<-\beta \\
& \text { if }-\frac{3}{2} \leq \beta<0 \\
& \text { or } \delta_{1}<\delta<\frac{3}{2} \\
& \text { if } \beta<-\frac{3}{2}
\end{aligned}
$$

$$
\begin{aligned}
& \delta_{2}<\delta<-\beta \\
& \text { if }-2 \leq \beta<0 \\
& \text { or } \delta_{2}<\delta<2 \\
& \text { if } \beta<-2 \\
& \delta_{4}<\delta<\frac{3}{2}-\beta \\
& \text { if }-\frac{1}{2} \leq \beta<0 \\
& \text { or } \delta_{4}<\delta<2 \\
& \text { if } \beta<-\frac{1}{2}
\end{aligned}
$$

No stable point

No stable point

No stable point $\delta_{3}<\delta<-\beta$

if $-3 \leq \beta<0$

or $\delta_{3}<\delta<3$

if $\beta<-3$

$\delta_{5}<\delta<\frac{3}{2}-\beta$

if $-\frac{3}{2} \leq \beta<0$

or $\delta_{5}<\delta<3$

if $\beta<-\frac{3}{2}$

$\delta_{6}<\delta<2-\beta$

if $-1 \leq \beta<0$

or $\delta_{6}<\delta<3$

if $\beta<-1$ sibilities are allowed if the equation of state parameters of DM and ELKO field are of radiation or ultrarelativistic type. Another interesting aspect that follows from Tables 1, 2, 3 is that there are no stable fixed points if $\omega_{\phi}>\omega_{m}$.

For the last interaction, namely $Q_{1}=\beta\left(v^{2}-y^{2}\right)$, we have the opposite. The conditions for stable fixed points in order to satisfy the present stage of acceleration are given by negative $\beta$ and positive $\delta$ parameters. The equation of state parameter for the ELKO field must be of radiation $\left(\omega_{\phi}=1 / 3\right)$ or ultrarelativistic $\left(\omega_{\phi}=1\right)$ type. The case $\omega_{\phi}=-1$ does not present stable fixed points. Negative values of $\beta$ mean the decay of DM particles into ELKO field. Such conditions also could alleviate the cosmological coincidence problem, and the equation of state parameter of ELKO field is not of exotic type. The inflationary phase cannot be driven for this interaction too. Other possibilities are allowed if the equation of state parameters of DM and ELKO field are of radiation or ultrarelativistic type. Another interesting aspect that follows from Table IV is that, contrary to the previous cases, there are no stable fixed points if $\omega_{\phi}<\omega_{m}$.

In such an analysis the interaction $Q$ must be proportional to the variable $x$, otherwise the relations among the parameters must be very restrictive. But such condition is not so strong, since the variable $x$ is proportional to $\dot{\phi}$, which characterizes a time dependence of the field $\phi$, and it is reasonable for an interacting theory. Notice that all the interactions studied are proportional to $\dot{\phi}^{2}$. For all the interactions analyzed here there are conditions of stability in order to alleviate the cosmic coincidence problem. Such kinds of interactions and conditions on the parameters $\beta$ and $\delta$ open possibilities for future searches concerning the interaction between DM and ELKO field for specific potentials satisfying the conditions presented in the tables.

Finally, a general theory that includes torsion in ELKO cosmology is of great interest, and in fact it may be the one responsible for the late time acceleration of the universe $[69,70]$. From a dynamical system approach the system of dynamical equation should be rewritten for new variables, certainly with some interesting consequences, but this is left for future.

Acknowledgments SHP is grateful to CNPq - Conselho Nacional de Desenvolvimento Científico e Tecnológico, Brazilian research agency, for the financial support, under process number 477872/2010-7. JFJ is grateful to Unesp - Câmpus Guaratinguetá and to SHP for hospitality and facilities. We would like to thank the referee for the suggestions and useful comments, especially in which the general treatment including torsion is concerned.

Open Access This article is distributed under the terms of the Creative Commons Attribution License which permits any use, distribution, and reproduction in any medium, provided the original author(s) and the source are credited.

Funded by $\mathrm{SCOAP}^{3}$ / License Version CC BY 4.0.

\section{References}

1. A.G. Riess et al., Observational evidence from Supernovae for an accelerating universe and a cosmological constant. Astron. J. 116, 1009 (1998)

2. S. Perlmutter et al., Measurements of $\Omega$ and Lambda from 42 high-redshift Supernovae. Astrophys. J. 517, 565 (1999) 
3. P. Astier et al., The Supernova Legacy Survey: measurement of $\Omega_{M}, \Omega_{\Lambda}$ and $\mathrm{w}$ from the first year data set. Astron. Astrophys. 447, 31 (2006)

4. A.G. Riess et al., New Hubble Space Telescope discoveries of Type Ia Supernovae at $z$ 1: narrowing constraints on the early behavior of dark energy. Astrophys. J. 659, 98 (2007)

5. E. Komatsu et al., Seven-year Wilkinson microwave anisotropy probe (WMAP) observations: cosmological interpretation. Astrophys. J. Suppl. 192, 18 (2011)

6. D. Larson et al., Seven-year Wilkinson microwave anisotropy probe (WMAP *) observations: power spectra and WMAP-derived parameters. Astrophys. J. Suppl. 192, 16 (2011)

7. T. Padmanabhan, Cosmological constant - the weight of the vacuum. Phys. Rep. 380, 235 (2003)

8. P.J.E. Peebles, B. Ratra, The cosmological constant and dark energy. Rev. Mod. Phys. 75, 559 (2003)

9. M. Davis, G. Efstathiou, C.S. Frenk, D.S.M. White, The evolution of large-scale structure in a universe dominated by cold dark matter. Astrophys. J. 292, 371 (1985)

10. M. Taoso, G. Bertone, A. Masiero, Dark matter candidates: a tenpoint test. JCAP 03, 022 (2008)

11. G. Bertone, Particle Dark Matter: Observations, Models and Searches (Cambridge University Press, UK, 2010)

12. R. Bernabei et al., First results from DAMA/LIBRA and the combined results with DAMA/NaI. Eur. Phys. J. C 56, 333 (2008)

13. J.A.S. Lima, Alternative dark energy models: an overview. Braz. J. Phys. 34, 194 (2004)

14. V. Sahni, A. Starobinsky, Reconstructing dark energy. Int. J. Mod. Phys. D 15, 2105 (2006)

15. E.J. Copeland, M. Sami, S. Tsujikawa, Dynamics of dark energy. Int. J. Mod. Phys. D 15, 1753 (2006)

16. E. Elizalde, S. Nojiri, S.D. Odintsov, P. Wang, Dark energy: vacuum fluctuations, the effective phantom phase, and holography. Phys. Rev. D 71, 103504 (2005)

17. F.C. Carvalho et al., Scalar-field-dominated cosmology with a transient acceleration phase. Phys. Rev. Lett. 97, 081301 (2006)

18. L.R. Abramo, N. Pinto-Neto, Stability of phantom k-essence theories. Phys. Rev. D 73, 063522 (2006)

19. T. Matos, F.S. Guzmán, L.A. Ureña-López, Scalar field as dark matter in the universe. Class. Quantum Gravity 17, 1707 (2000)

20. F.S. Guzmán, T. Matos, Scalar fields as dark matter in spiral galaxies. Class. Quantum Gravity 17, 9 (2000)

21. A. Suárez, V. Robles, T. Matos, A review on the scalar field/BoseEinstein condensate dark matter model. Astrophys. Space Sci. 9, 38 (2013)

22. T. Matos, A. Vázquez-González, J. Magaña, $\phi^{2}$ as dark matter. Mon. Not. R. Astron. Soc. 393, 1359 (2009)

23. L.A. Ureña-López, Bose-Einstein condensation of relativistic scalar field dark matter. JCAP 014, 0901 (2009)

24. L.A. Ureña-López, M.J. Reyes-Ibarra, On the dynamics of a quadratic scalar field potential. Int. J. Mod. Phys. D 18, 621 (2009)

25. V.H. Robles, T. Matos, Flat central density profile and constant dark matter surface density in galaxies from scalar field dark matter. Mon. Not. R. Astron. Soc. 422, 282 (2012)

26. J. Magaña, T. Matos, A. Suárez, F.J. Sánchez-Salcedo, Structure formation with scalar field dark matter: the field approach. JCAP 10, 003 (2012)

27. J. Magaña, T. Matos, V. Robles, A. Suárez, A brief review of the scalar field dark matter model (2012). arXiv:1201.6107

28. U.D. Jentschura, B.J. Wundt, From generalized dirac equations to a candidate for dark energy. ISRN High Energy Phys. 2013, 21 (2013)

29. O.V. Babourova, B.N. Frolov, R.S. Kostkin, Dirac's scalar field as an effective component of the dark energy and an evolution of the cosmological "constant" (2011). arXiv:1102.2901
30. Z.-Y. Sun, Y.-G. Shen, Dark energy cosmology with spinor field. Int. J. Theor. Phys. (2006). doi:10.1007/s10773-006-9128-x

31. S. Das, P.S. Corasaniti, J. Khoury, Super-acceleration as signature of dark sector interaction. Phys. Rev. D 73, 083509 (2006)

32. C. Feng, B. Wang, Y.G. Gong, R.K. Su, Testing the viability of the interacting holographic dark energy model by using combined observational constraints. JCAP 005, 0709 (2007)

33. G.M. Kremer, Dark energy interacting with neutrinos and dark matter: a phenomenological theory. Gen. Relativ. Gravit. 39, 965 (2007)

34. C.G. Boehmer, G. Caldera-Cabral, R. Lazkoz, R. Maartens, Dynamics of dark energy with a coupling to dark matter. Phys. Rev. D 78, 023505 (2008)

35. W. Zimdahl, D. Pavón, L.P. Chimento, Interacting quintessence. Phys. Lett. B 521, 133 (2001)

36. L.P. Chimento, A.S. Jakubi, D. Pavón, W. Zimdahl, Interacting quintessence solution to the coincidence problem. Phys. Rev. D 67, 083513 (2003)

37. B. Wang, Y.G. Gong, E. Abdalla, Transition of the dark energy equation of state in an interacting holographic dark energy model. Phys. Lett. B 624, 141 (2005)

38. B. Wang, C.Y. Lin, E. Abdalla, Constraints on the interacting holographic dark energy model. Phys. Lett. B 637, 357 (2006)

39. B. Wang, J. Zang, C.Y. Lin, E. Abdalla, S. Micheletti, Interacting dark energy and dark matter: observational constraints from cosmological parameters. Nucl. Phys. B 778, 69 (2007)

40. E. Abdalla, L.R. Abramo, L. Sodré, B. Wang, Signature of the interaction between dark energy and dark matter in galaxy clusters. Phys. Lett. B 673, 107 (2009)

41. B. Wang, C.Y. Lin, D. Pavón, E. Abdalla, Thermodynamical description of the interaction between dark energy and dark matter. Phys. Lett. B 662, 1 (2008)

42. Z.K. Guo, N. Ohta, S. Tsujikawa, Probing the coupling between dark components of the universe. Phys. Rev. D 76, 023508 (2007)

43. M. Quartin, M.O. Calvão, S.E. Jorás, R.R.R. Reis, I. Waga, Dark interactions and cosmological fine-tuning. JCAP 0805, 007 (2008)

44. S.H. Pereira, J.F. Jesus, Can dark matter decay in dark energy? Phys. Rev. D 79, 043517 (2009)

45. D.V. Ahluwalia-Khalilova, D. Grumiller, Dark matter: a spin onehalf fermion field with mass dimension one? Phys. Rev. D 72, 067701 (2005)

46. D.V. Ahluwalia-Khalilova, D. Grumiller, Spin half fermions with mass dimension one: theory, phenomenology, and dark matter. JCAP 07, 012 (2005)

47. D.V. Ahluwalia, On a local mass dimension one Fermi field of spin one-half and the theoretical crevice that allows it (2013). arXiv: $1305.7509 \mathrm{v} 1$ [hep-th]

48. C.G. Boehmer, The Einstein-Cartan-Elko system. Ann. Phys. 16, 38 (2007)

49. C.G. Boehmer, J. Burnett, Dark spinors with torsion in cosmology. Phys. Rev. D 78, 104001 (2008)

50. C.G. Boehmer, D.F. Mota, CMB anisotropies and inflation from non-standard spinors. Phys. Lett. B 663, 168 (2008)

51. L. Fabbri, The most general cosmological dynamics for ELKO matter fields. Phys. Lett. B 704, 255 (2011)

52. C.G. Boehmer, The Einstein-Elko system—can dark matter drive inflation? Ann. Phys. 16, 325 (2007)

53. C.G. Boehmer, Dark spinor inflation-theory primer and dynamics. Phys. Rev. D 77, 123535 (2008)

54. C.G. Boehmer, J. Burnett, D.F. Mota, D.J. Shaw, Dark spinor models in gravitation and cosmology. JHEP 07, 053 (2010)

55. D. Gredat, S. Shankaranarayanan, Modified scalar and tensor spectra in spinor driven inflation. JCAP 01, 008 (2010)

56. A. Basak, J.R. Bhatt, Lorentz invariant dark-spinor and inflation. JCAP 06, 011 (2011) 
57. C.G. Boehmer, J. Burnett, Dark energy with dark spinors. Mod. Phys. Lett. A 25, 101 (2010)

58. R. da Rocha, A.E. Bernardini, J.M. Hoff da Silva, Exotic dark spinor fields. JHEP 04, 110 (2011)

59. R. da Rocha, J.M. Hoff da Silva, ELKO spinor fields: Lagrangians for gravity derived from supergravity. Int. J. Geom. Methods Mod. Phys. 6, 461 (2009)

60. R. da Rocha, J.M. Hoff da Silva, From Dirac spinor fields to ELKO. J. Math. Phys. 48, 123517 (2007)

61. J.M. Hoff da Silva, R. da Rocha, From Dirac action to ELKO action. Int. J. Mod. Phys. A 24, 3227 (2009)

62. J.M. Hoff da Silva, S.H. Pereira, Exact solutions to Elko spinors in spatially flat Friedmann-Robertson-Walker spacetimes. JCAP 03, 009 (2014)

63. M. Dias, F. de Campos, J.M. Hoff da Silva, Exploring Elko typical signature. Phys. Lett. B 706, 352 (2012)

64. A. Alves, F. de Campos, M. Dias, J.M. Hoff da Silva, Searching for Elko dark matter spinors at the CERN LHC (2014). arXiv: $1401.1127 \mathrm{v} 2$

65. H. Wei, Spinor dark energy and cosmological coincidence problem. Phys. Lett. B 695, 307 (2011)
66. A. Basak, J. R. Bhatt, S. Shankaranarayanan, K.V.P. Varma, Attractor behaviour in ELKO cosmology. JCAP 04, 025 (2013)

67. H.M. Sadjadi, On coincidence problem in ELKO dark energy model. Gen. Relativ. Gravit. 44, 2329 (2012)

68. S.H. Pereira, A.P. Santos Souza, J. M. Hoff da Silva, Some remarks on the attractor behaviour in ELKO cosmology. JCAP 08, 020 (2014)

69. L. Fabbri, Conformal gravity with the most general ELKO fields. Phys. Rev. D 85, 047502 (2012)

70. L. Fabbri, S. Vignolo, A modified theory of gravity with torsion and its applications to cosmology and particle physics. Int. J. Theor. Phys. 51, 3186 (2012)

71. S.H. Strogatz, Nonlinear Dynamics and Chaos (Perseus Books Publishing, Massachusetts, 1994)

72. P.A.R. Ade et al., Planck 2013 results. XVI. Cosmological parameters. Astron. Astrophys. (2013). arXiv:1303.5076 